\title{
Discours
}

Revue de linguistique, psycholinguistique et

informatique. A journal of linguistics, psycholinguistics and computational linguistics

$5 \mid 2009$

Varia

\section{Rabatel, A. 2008. Homo Narrans. Pour une analyse énonciative et interactionnelle du récit. Limoges : Lambert-Lucas. 2 vol., 689 p.}

\section{Laurent Perrin}

\section{OpenEdition}

\section{Journals}

Édition électronique

URL : http://journals.openedition.org/discours/7451

DOI : $10.4000 /$ discours.7451

ISSN : 1963-1723

\section{Éditeur :}

Laboratoire LATTICE, Presses universitaires de Caen

\section{Référence électronique}

Laurent Perrin, «Rabatel, A. 2008. Homo Narrans. Pour une analyse énonciative et interactionnelle du récit. Limoges : Lambert-Lucas. 2 vol., 689 p. », Discours [En ligne], 5 | 2009, mis en ligne le 12 juillet 2009, consulté le 22 septembre 2020. URL : http://journals.openedition.org/discours/7451 ; DOI : https://doi.org/10.4000/discours.7451

\section{(c) (i) (3) $\Theta$}

Discours est mis à disposition selon les termes de la licence Creative Commons Attribution - Pas d'Utilisation Commerciale - Pas de Modification 4.0 International. 


\title{
Rabatel, A. 2008. Homo Narrans. Pour une analyse énonciative et interactionnelle du récit. Limoges : Lambert-Lucas. 2 vol., 689 p.
}

\author{
Laurent Perrin
}

Université Paul Verlaine - Metz, CELTED, EA 3474

1 Homo narrans est une étude de grande ampleur, très savante et consistante, consacrée à l'expression des perceptions et opinions dans le langage et par le langage. Il s'agit d'une réflexion approfondie et élargie, au carrefour de la linguistique, de l'analyse du discours et de la narratologie (qui n'hésite pas en outre à s'aventurer du côté de la sociologie, de l'anthropologie, des sciences cognitives, de la philosophie du langage), visant à saisir comment le sujet s'inscrit dans le langage, comment plus précisément le langage exprime et instrumentalise la perception, l'opinion, la subjectivité associée à son énonciation, sous la forme de différents points de vue représentés. L'ouvrage donne une cohérence d'ensemble aux recherches d'Alain Rabatel, disséminées jusqu'ici dans plusieurs dizaines d'articles et dans trois ouvrages préalables. L'approche élaborée est bien trop vaste, ses ramifications trop diversifiées et sinueuses, pour espérer en rendre compte et surtout leur rendre justice en quelques pages. Je me contenterai donc de considérations générales et terminerai par des remarques très fragmentaires, relatives à quelques aspects de l'analyse au plan théorique.

$$
* * *
$$

2 L'approche élaborée s'inscrit dans une tradition sémantique énonciative et dialogique (ou polyphonique), qui conçoit le sens des énoncés comme fondamentalement réflexif, comme qualifiant l'emploi, l'énonciation des formes linguistiques, les points de vue qui s'y rapportent, ceci jusque dans l'acte même qui consiste, le cas échéant, à décrire une réalité, à raconter une histoire par exemple. Le langage, dans cette optique, ne consiste pas simplement à décrire, à représenter des états de chose (des mondes, des histoires), mais consiste à se représenter lui-même, sui-référentiellement en quelque sorte, comme l'expression de tel ou tel point de vue. La place centrale de la notion de réflexivité (et de pré-réflexivité) dans les analyses d'Alain Rabatel, fonde la perspective énonciative et dialogique revendiquée. De la notion de réflexivité dépend non seulement celle de point de vue représenté, mais toute la dimension dialogique, interdiscursive ou interactionnelle, attribuée aux énoncés dans cette étude. Homo narrans relève d'une tradition sémantique issue de Bakhtine (1929) et de Bally (1932), de Benveniste (1966), relayée notamment par Banfield (1995), Authier-Revuz (1995), jusqu'à Ducrot (1984) qui consacre la réflexivité du sens au plan sémantique généralisé. 
3 Mais le rapprochement, avec Ducrot en particulier, s'arrête là, car Homo narrans n'est pas une linguistique du discours idéal, tel qu'il est idéalisé dans la langue, mais une linguistique du discours ordinaire, fût-il narratif et littéraire. Contrairement à Ducrot et aux approches qui s'en inspirent, Homo narrans n'est pas une théorie sémantique des représentations énonciatives et polyphoniques en langue, mais d'abord et avant tout une approche empirique des textes, en particulier des récits. Le refus de se couper de l'expérience du texte, de l'énoncé authentique - qu'il s'agisse d'une œuvre littéraire ou de n'importe quelle séquence discursive - caractérise toute l'entreprise d'Homo narrans, et en fait aussi toute la force et l'intérêt. Tournée vers l'interprétation et l'herméneutique, la réflexion linguistique ne se replie pas ici sur la langue et sur elle-même; elle se déploie dans la lecture des textes et s'applique à en révéler la singularité. Très loin des entreprises de réduction «ad absurdum » qu'appréhendait Jakobson (1963, 213), qui confinent l'analyse à telle ou telle idéalisation linguistique plus ou moins formalisée sans toujours parvenir à la mettre en œuvre au plan empirique, loin même de la facilité si fréquente qui consiste à se servir d'exemples authentiques pour étayer une hypothèse, Homo narrans fait l'inverse : il met l'analyse au service du texte et de la lecture. Cette dernière n'est pas là pour exemplifier, pour illustrer, mais pour être ellemême éclairée par l'analyse et la réflexion.

Une telle posture impose de transcender le plan linguistique des marques associées aux mots et aux phrases afin d'appréhender les séquences discursives dans toute la complexité de leur contexte textuel, générique ou scénographique, situationnel, interactionnel. Il s'agit dès lors non seulement de franchir la barrière de la phrase ou tout au moins de la portée locale des relations grammaticales, afin d'appréhender l'organisation discursive des énoncés au plan narratif, argumentatif, ou interactionnel. Mais il s'agit surtout d'opérer ce transfert sous l'angle énonciatif et dialogique, plutôt que sous l'angle d'une cohérence purement descriptive et référentielle, et selon un appareillage de règles immanentes relatives au récit, au raisonnement logique, ou encore à la conversation. On peut être reconnaissant à Homo narrans de franchir ce pas avec succès. L'ouvrage explore incontestablement de nombreuses pistes prometteuses, et élabore au passage différents concepts pragmatiques opératoires.

5 Les observations de Bakhtine sur le dialogisme et la polyphonie, les développements qui en sont issus en analyse du discours et en linguistique textuelle ces dernières décennies en France, tout comme l'influence des analyses conversationnelles et autres sociologies de la communication anglosaxonnes, ont ouvert la voie à Homo narrans, qui se conçoit comme une entreprise de reformulation énonciative et interactionnelle des principaux enjeux de la narratologie, de ce qui a trait aux voix et aux modes narratifs en particulier chez Genette (1972). Homo narrans se veut une approche énonciative du récit, non comme un texte préstructuré tourné vers une histoire (au sens des structuralistes), mais comme une narration, c'est-à-dire un 
comportement propre au langage humain, fondé sur la notion de point de vue représenté, sur la conception énonciative et dialogique qui s'y rapporte.

L'approche concerne non seulement les récits, mais l'ensemble des formes de narration et finalement toute forme de discours. Alain Rabatel insiste à plusieurs reprises sur le fait que s'il s'intéresse en particulier au récit sous l'angle des points de vue des personnages qu'il met en jeu, de leur relation à la voix du narrateur et aux instances virtuelles qui s'y trouvent associées (comme celle, par exemple, du lecteur, ou encore de la doxa, d'un on-locuteur), ses analyses concernent au même titre l'articulation des instances associées au locuteur et aux différents énonciateurs dans toute séquence discursive, qu'elle soit ou non narrative au bout du compte. Homo narrans est une narratologie qui déborde de toute part du récit et de la narration pour envahir le discours délibératif et l'argumentation dans n'importe quel contexte.

L'ouvrage se présente sous la forme de deux forts volumes (en tout près de 700 pages), qui correspondent à deux tentatives d'appréhension de la notion de point de vue représenté, comme fait narratif d'abord, parmi d'autres formes de dialogisme ensuite. Chacun des volumes aborde le point de vue représenté sous un angle un peu différent, même si les deux se recoupent très largement; et chacun s'aventure ensuite dans des analyses de texte très riches et diversifiées. Au plan théorique, le premier volume est centré sur les propriétés énonciatives du point de vue représenté dans le récit, que le second se réapproprie comme celles d'une forme parmi d'autres de relation dialogique ou polyphonique. La progression du raisonnement d'un volume à l'autre ne saute pas forcément aux yeux, car les deux s'intéressent au point de vue représenté comme forme d'énonciation narrative dialogique ou polyphonique. On se demande même parfois ce qui justifie d'aborder telle ou telle étude de cas dans le premier volume plutôt que dans le second ou inversement.

8 En tant que propriété du discours narratif dans le premier volume, le point de vue représenté implique un narrateur s'exprimant par le biais de la perception, de la compréhension, de la voix même parfois de ses personnages (mais aussi de la doxa, d'une collectivité, d'un lecteur fictif auquel il s'adresse). Le point de vue représenté est opposé à deux autres formes de médiation du point de vue que sont respectivement le point de vue raconté d'une part, et asserté d'autre part. Il s'agit d'abord de saisir ce qui oppose un point de vue représenté, c'est-à-dire en quelque sorte rapporté dans un récit (en un sens technique qui sera défini plus précisément dans le second volume), à un point de vue simplement raconté, c'est-à-dire impliqué sous forme actantielle, mais sans être exprimé, c'est-à-dire sans occuper d'espace séquentiel ni recevoir de rôle énonciatif. Vue sous cet angle, «la spécificité du point de vue représenté [...] est de construire un espace énonciatif dans lequel la subjectivité des perceptions de l'énonciateur peut se donner libre cours » précise Alain Rabatel 
(p. 102). Mais d'autre part, la notion de point de vue représenté s'établit aussi par opposition à un point de vue simplement asserté, c'est-à-dire assumé directement par le locuteur, notamment en contexte argumentatif, ou encore par le locuteur d'un énoncé rapporté au style direct. Dans ce cas, le point de vue est bel et bien exprimé et énoncé, mais sans être représenté, du moins au sens technique.

9 Cette seconde opposition, entre point de vue représenté et asserté, pourtant tout à fait essentielle, est malheureusement laissée un peu en suspens dans cette étude. La notion de point de vue asserté ne sera pas exploitée par la suite dans les analyses de textes, et pas davantage reprise au plan théorique dans le second volume, centré sur la notion de discours représenté. Que faire en effet de la notion de point de vue asserté dans le cadre de ce qui oppose le discours au point de vue représenté ? Que faire notamment de cette notion pour rendre compte du discours direct? Le discours rapporté au style direct (et direct libre) implique-t-il un point de vue asserté (par le personnage) dans le cadre d'un discours représenté (par le narrateur) ? Et que devient ensuite ce point de vue asserté dans le cas du style indirect libre et des formes mixtes, des formes de modalisations autonymiques? Étant donné que ces dernières ne s'opposent que graduellement au style direct, ne consistent-elles pas elles aussi à intégrer, sous forme affaiblie peut-être, un point de vue asserté dans le cadre d'un discours ou d'un point de vue représenté ? Et qu'en est-il au style indirect, et en cas de simple reformulation? Le point de vue étranger est-il alors purement représenté (par le narrateur) sans être nullement asserté (par le personnage) ?

10 Au-delà de ces difficultés, la notion de point de vue asserté s'applique-t-elle par ailleurs à l'engagement du narrateur en tant que tel, c'est-à-dire en tant que responsable d'un énoncé narratif impliquant le point de vue d'un personnage ? Il est intéressant d'observer à ce sujet que les exemples de points de vue assertés en (37) et (38), assimilés à «d'authentiques points de vue du narrateur » (p. 107), ne sont en fait nullement narratifs, en l'absence d'imputation des points de vue exprimés à un personnage. Même dans un récit, le narrateur redevient un simple locuteur assertant un point de vue lorsqu'il cesse de représenter celui d'un personnage. Mais qu'advient-il de cette assertion dans le cas d'un point de vue représenté ? Le point de vue représenté d'un personnage peut-il être simultanément asserté par le narrateur en certaines circonstances? Et qu'en est-il en cas d'effacement énonciatif et d'argumentation indirecte ? Et en cas de co-énonciation ? Toutes ces questions ne sont pas vraiment clarifiées, ce qui laisse un peu sur sa faim en ce qui concerne le cadrage macro-théorique de l'ouvrage.

11 Le premier volume d'Homo narrans s'emploie en revanche à analyser très finement l'organisation des points de vue représentés dans le discours narratif, leur relation au narrateur, aux personnages, et aux autres instances énonciatives impliquées, les stratégies qu'elles déclenchent en terme d'argumentation indirecte, de co-énonciation ou de sur/sous-énonciation, tout 
ceci en fonction de différents ensembles de marques linguistiques (présentatifs, connecteurs, temps verbaux, ordre des mots) et dans différentes œuvres (de la bible à quelques auteurs majeurs des $\mathrm{XIX}^{\mathrm{e}}$ et $\mathrm{XX}^{\mathrm{e}}$ siècles). La grande force d'Homo narrans, sur laquelle nous ne nous attarderons pas, mais à laquelle on ne peut que rendre hommage, tient à sa faculté d'articuler les observations de détails linguistiques ou stylistiques à des enjeux interprétatifs de premier plan.

12 Quant au second volume, il reprend la réflexion du précédent en cherchant cette fois à clarifier la relation entre discours et point de vue représenté, notamment au style direct et indirect, libre ou lié à un verbe régisseur, sans parler des formes mixtes, des îlots textuels, et autres effets dialogiques plus ou moins marqués (nous n'entrerons pas ici en matière sur les avantages et les inconvénients associés à la notion de discours ou de point de vue représenté, plutôt que rapporté, pas plus que sur d'autres distinctions discutables, comme celle entre dialogisme et polyphonie). Suite à deux chapitres consacrés au dialogisme et à la polyphonie selon Bakhtine, et à la sous-évaluation narratologique de cette dimension chez Genette, l'objectif est désormais de saisir ce qui fait du point de vue représenté une forme particulière de dialogisme, parmi différentes formes apparentées, assimilées à du discours représenté. Une telle opposition mobilise un dispositif théorique complexe jouant sur plusieurs paramètres graduels de dissociation entre parole et pensée, perception, mais aussi d'effacement énonciatif, et de variation d'intensité réflexive ou mimétique de la représentation. Force est pourtant de constater, malgré la finesse de certaines observations, que la longue discussion sur les oppositions de formes, initiée au chapitre 2 à propos des degrés de mimèsis chez Genette, et poursuivie jusqu'au chapitre 4 et même au-delà, ne clarifie pas forcément les choses par rapport aux nombreuses tentatives préalables de classification polarisée des formes du discours rapporté sous différents angles.

13 Comme d'autres prédécesseurs parfois illustres, Alain Rabatel fait peut-être ici les frais de son approche empirique des phénomènes, qui le conduit à appréhender globalement ce qui distingue, par nature, le discours et la perception, la pensée (dont atteste la distinction entre verba dicendi, sentiendi, putandi), et ce qui oppose formellement et fonctionnellement par ailleurs les formes de discours ou de pensée représentée, ce qui oppose le style direct au style indirect notamment, ou encore le style indirect libre au point de vue représenté. Sans du tout entrer ici en discussion sur d'éventuelles solutions susceptibles de remédier à ces difficultés, on peut encore relever qu'Alain Rabatel fait aussi sur ces questions les frais théoriques d'une certaine impuissance de la sémantique (pour ne pas dire une impuissance certaine) à tirer toutes les conséquences de ce qui oppose (et souvent superpose) à l'intérieur du sens des énoncés, non seulement modus et dictum, mais ce qui est montré et ce qui est dit, représenté propositionnellement, et dans ce qui est montré, ce qui est indiciel (indexical, énonciatif) et ce qui est iconique (mimétique), et dans ce qui est iconique... Tant que ces distinctions sémantiques ne seront pas clairement établies et prises en compte, les 
oppositions entre différentes formes de discours représenté seront condamnées à se mordre la queue.

14 Mais tout cela, encore une fois, n'enlève rien au mérite de l'ouvrage, qui est précisément de s'arracher à ces difficultés, de surmonter l'obstacle linguistique, afin d'élaborer différents concepts pragmatiques opératoires (de sur-énonciation et sous-énonciation, d'effacement énonciatif, d'argumentation indirecte) susceptibles de fonder une herméneutique énonciative applicable non seulement au récit, mais à d'autres formes de discours, notamment au monologue intérieur, à l'idiolecte, à l'autocitation. La véritable force d'Homo narrans, au-delà des difficultés théoriques que rencontre inévitablement toute tentative d'appréhension du sens à ce niveau de généralité, c'est ce souffle impressionnant qui traverse la littérature, le langage et la pensée, laissant ici ou là le lecteur un peu étourdi et dérouté, mais souvent éclairé et enrichi. Ce n'est pas rien.

\section{BIBLIOGRAPHIE}

Authier-Revuz, J. 1995. Ces mots qui ne vont pas de soi. Boucles réflexives et non-coïncidences du dire. Paris : Larousse.

BALly, CH. 1965/1932. Linguistique générale et linguistique française. Berne : Francke.

BAnfiEld, A. 1995/1982. Phrases sans parole. Théorie du récit et du style indirect libre. Paris : Le Seuil.

BAKHtine, M. 1970/1929. Le poétique de Dostoïevski. Lausanne: L’Âge d'Homme.

Benveniste, E. 1966. Problèmes de linguistique générale. Paris : Gallimard.

Ducrot, O. 1984. Le dire et le dit. Paris : Minuit.

GenetTe, G. 1972. Figures III. Paris : Le Seuil.

JAKOBSON, R. 1963. Essai de linguistique générale, vol. 1. Paris : Le Seuil.

\section{Pour citer cet article}

Laurent Perrin, «Rabatel, A. 2008. Homo Narrans. Pour une analyse énonciative et interactionnelle du récit. Limoges : Lambert-Lucas. 2 vol., 689 p. », Discours, 5 | 2009, [En ligne], mis en ligne le 12 juillet 2009. URL : http://discours.revues.org/index 7451.html. 